\title{
China, Estados Unidos y 5G: Capitalismo de Vigilancia, Geopolítica y Geoestrategia
}

\author{
China, the United States and 5G: Surveillance Capitalism, \\ Geopolitics and Geostrategy
}

Natalia Chaparro Betancourt ${ }^{1^{*}}$; Vladimir Osorio Isaza ${ }^{2}$ y Adolfo Ernesto Sandoval Perdomo ${ }^{3}$

(1) Universidad Militar Nueva Granada - UMNG, Bogotá - Colombia, nataliachaparro.3098@gmail.com

(2) Escuela de Inteligencia y Contrainteligencia “BG. Ricardo Charry Solano” - ESICI, Bogotá - Colombia, vsaza35@ gmail.com

(3) Escuela de Inteligencia y Contrainteligencia "BG. Ricardo Charry Solano" - ESICI, Bogotá - Colombia, adolfo_ sandoval@javeriana.edu.co

* Autor a quien se dirige la correspondencia

\section{Resumen}

China y Estados Unidos se encuentran en una disputa internacional por liderar la implementación de la tecnología 5G, y las acciones de Estados Unidos en contra de la empresa Huawei son una muestra de ello. Más allá de un conflicto comercial, es un problema de seguridad nacional en el que está en juego la seguridad de la información. En este artículo se formula la pregunta ¿cuáles intereses geopolíticos están en juego detrás de la instalación de las redes $5 \mathrm{G}$ y por qué existe tanta reticencia a que sea una compañía china la que lidere este mercado? Para responder este cuestionamiento se realizó un análisis coyuntural de hechos a partir de artículos de prensa y de trabajos de investigación académica. Se concluye que estas potencias están inmersas en una competencia por el monopolio de la explotación y almacenamiento de Big Data, por lo que el desarrollo de infraestructura $5 \mathrm{G}$ se convierte en un factor estratégico que podría modificar el status quo en el sistema internacional.

Palabras clave: 5G; Geopolítica; Seguridad nacional; Big Data; China; Estados Unidos.

\section{Abstract}

China and the United States are in an international dispute to lead the implementation of $5 \mathrm{G}$ technology, and the actions of the United States against Huawei are a sign of this. Beyond a commercial problem, it is a national security problem where information security is at stake. This article asks the question: What geopolitical interests are at stake behind the installation of $5 \mathrm{G}$ networks and why is there so much reluctance for a Chinese company to lead this market? To answer this question, a situational analysis of press articles and research papers was conducted. We conclude that these powers are immersed in a competition for the monopoly of the exploitation and storage of big data, so the development of $5 G$ infrastructure becomes a strategic factor that could change the status quo in the international system.

Keywords: 5G; Geopolitics; National security; Big Data; China; United States. 


\section{Introducción}

El acceso y uso preferente de los flujos de información siempre han sido un asunto primordial para los Estados, dado que resultan ser una herramienta estratégica para favorecer sus intereses a nivel nacional e internacional. Esto se puede observar en varios momentos de la historia, por ejemplo, la Conquista española sobre el Imperio Inca no podría haberse dado sin el asesinato selectivo de los chasquis (mensajeros personales del inca); sin ellos, el Imperio quedó desposeído de la información necesaria para defenderse, ocasionando su caída (Gavaldá, 2020). De igual forma, en la guerra de Vietnam, la red de túneles de la que disponía el Vietcom ${ }^{1}$ fue indispensable para darles la victoria, ya que esta no servía únicamente como refugio, sino que era a su vez el medio para reportar la información clave hacia los mandos de la resistencia, que en perfecta coordinación podían dirigir ataques contra el Ejército norteamericano (García, 2018).

En la era del internet, la información está lejos de perder su importancia. En un mundo en el que todas las personas publican sus vidas y datos a cambio de un acceso a la red, los datos ya no guardan el mismo hermetismo que antes, sin embargo, esto no le ha restado valor a la información. Quien controle los canales por donde circula la inmensa cantidad de datos y pueda hacerse garante de los flujos de información tiene en su poder la ventaja de saber con antelación qué contienen esos datos, y con ello darle el uso comercial o estratégico que le favorezca. Es en este punto donde la tecnología de quinta generación $(5 G)$ toma relevancia en el sistema internacional. China y Estados Unidos están inmersos en una carrera por alcanzar la supremacía tecnológica que, a su vez, se convierte en un campo de enfrentamiento geopolítico entre estas grandes naciones. El que logre primero el desarrollo de la infraestructura para la implementación de la $5 G$ tendrá un acceso privilegiado a la información, además de aumentar su esfera de influencia y con ello tener una ventaja a la hora de imponer sus intereses económicos, políticos y hasta culturales.

La economista y socióloga Shoshana Zuboff, de la Universidad de Harvard, ofrece un análisis fructífero desde lo que ella ha acuñado como capitalismo de vigilancia, en su libro homónimo "The age of surveillance capitalism", publicado en 2019 (Zuboff, 2019). A partir de su análisis y el seguimiento informativo de las tensiones entre las dos mayores potencias se indagarán, en este artículo, los límites e intereses más allá de lo que parece obvio en la disputa por la instalación de las redes $5 \mathrm{G}$; un tema muy comentado en medios de comunicación y la opinión pública, pero del que se ha investigado muy poco desde una perspectiva de seguridad de la información, siendo un limitante a la hora de abordar el tema.

\section{¿Qué es la tecnología 5G?}

La denominación $5 \mathrm{G}$ se refiere a la quinta generación de redes móviles que aumentará las velocidades de conexión de la red $4 \mathrm{G}$. A diferencia de las antiguas redes $1 \mathrm{G}$ y $2 \mathrm{G}$ de los primeros teléfonos móviles, que solo permitían hacer llamadas y el servicio de SMS (servicio de mensajes cortos), o de las redes 3G y $4 G$ que incorporaron la conexión a internet banda ancha y aumentaron la velocidad de conexión, permitiendo la reproducción de videos en tiempo real (Flores, 2019). 
Rodrigo Orellana, en su portal "digitaltrends" (2020), señala que la tecnología 5G tendrá tres características nuevas:

1. Banda ancha móvil mejorada: Tendrá el avance más significativo en cuanto a velocidad. Esta tecnología permitirá navegar hasta a $10 \mathrm{GBps}$ (gigabytes por segundo), 10 veces más rápido que las principales ofertas de fibra óptica del mercado $4 \mathrm{G}$.

2. Latencia confiable y baja: El tiempo de respuesta en la red se reducirá a 5 milisegundos, un periodo casi imperceptible para los seres humanos, pero muy significativo para mejorar la producción de componentes de reacción de muchos aparatos tecnológicos. Por ejemplo, a la hora de evaluar el funcionamiento de un vehículo autónomo, la minimización del tiempo de respuesta puede mejorar la seguridad tanto de los ocupantes como de cualquier circundante.

3. Conectividad masiva de dispositivos: Gracias a la mayor velocidad y disminución de latencia, se podrá aumentar el número de dispositivos conectados en tiempo real. El estándar indica que debería ser un millón por kilómetro cuadrado. Por lo que la 5G se convierte en una herramienta que va a facilitar el desarrollo de la inteligencia artificial (IA) $)^{2}$ y del internet de las cosas $(\mathrm{loT})^{3}$.

La tecnología 5G lleva consigo la promesa de revolucionar la industria y la forma en cómo se relacionan las personas. Aunque con la tecnología $3 G$ y $4 G$ ya se pueden desarrollar actividades de interconexión entre dispositivos y comunicación en tiempo real, es la combinación de velocidad, la latencia disminuida y el alcance que tiene la $5 \mathrm{G}$ la que puede desarrollar todas las capacidades de otras tendencias tecnológicas del momento, ofreciendo un fuerte impulso a los vehículos autónomos, drones, realidad virtual, cirugías teleasistidas y el internet de las cosas, que va a dar forma a las ciudades digitales $^{4}$ (Cheng, 2017).

Sin embargo, para la implementación de la $5 \mathrm{G}$ se necesitará de una nueva infraestructura tecnológica ${ }^{5}$. Grandes partes de la infraestructura de telecomunicaciones existentes deben actualizarse y ampliarse para admitir datos más rápidos. Sami Badri, analista de valores de Credit Suisse, señaló que el desarrollo para la implementación de la infraestructura $5 \mathrm{G}$ abarca varios sectores del mundo de las comunicaciones. Las primeras fases incluyen el despliegue de toda una infraestructura de red compuesta de antenas, radares y equipos de torre, además de la implementación del diseño de nuevos softwares para su funcionamiento. Se debe construir toda una nueva infraestructura que reemplazará la red anterior (Miller, 2019).

\footnotetext{
2 La Inteligencia artificial es el campo científico de la informática que se centra en la creación de programas y mecanismos que pueden mostrar comportamientos considerados inteligentes. Es decir, comportamientos que imitan las funciones cognitivas que los humanos asocian con otras mentes humanas, como percibir, razonar, resolver problemas etc. (Torra, 2020).

3 Se refiere a la interconexión digital de objetos cotidianos con el internet.

4 Son las ciudades que incorporan en la vida cotidiana las tecnologías de la información y de comunicaciones

5 La IT es el conjunto de elementos para el almacenamiento de información y procesamiento de datos. En ella se incluye el hardware (parte física para el desarrollo de una actividad tecnológica, como ordenadores, sensores, escáneres, Wi-fi etc.), el software (programas y sistemas que facilitan el funcionamiento de sistemas operativos y programas informáticos) y los diferentes servicios necesarios para optimizar la gestión interna y seguridad de información (Red Hat, s.f.).
} 


\section{La geopolítica del 5G: el debate actual}

En los últimos 10 años aparece la empresa Huawei en el escenario internacional. Se trata de una empresa tecnológica multinacional china, que proporciona equipos de telecomunicaciones y vende electrónica de consumo y teléfonos inteligentes. Esta firma domina el mercado de teléfonos móviles en China, y para el año 2020 se posicionó como la marca de teléfonos más vendida en el mundo; y a pesar de que sus ventas cayeron desde que Estados Unidos empezó a imponerles sanciones a nivel internacional, lograron vender 55.8 millones de teléfonos en el 2020 (la mayor parte en China), superando por primera vez a la marca Samsung (Agencia AFP, 2020). Su participación en el mercado global ha aumentado, pasó en el año 2018 de tener el 11,8\%, en el primer trimestre, a tener en el mismo periodo del año 2019 el 19\% y, de acuerdo con el reporte trimestral de IDC, fue la única compañía entre las tres primeras que reportó crecimiento en ese primer trimestre de 2019. Operacionalmente, mientras Apple tuvo una disminución del 30,2\% y Samsung del 8,1\%, Huawei tuvo un incremento del 50,3\%. Convirtiéndose en uno de los principales competidores de las dos marcas insignias de este mercado: Apple y Samsung (CNN, 2019).

En la actualidad Huawei lidera el campo de la infraestructura 5G. Para 2020 es el fabricante con más patentes de tecnología $5 \mathrm{G}$ en el mundo. De acuerdo con un reporte de las firmas de investigación Amplified y GreyB, a Huawei ya se le han concedido 952 de las 2.386 patentes manifestadas (40\% del total), convirtiéndose en pionero de la infraestructura 5G. Para febrero de 2018, con ayuda de Vodafone, lanzó el primer chip integral para estaciones-bases 5G (las instalaciones que permiten su comunicación), y hasta la fecha ha suscrito un total de 91 contratos comerciales $5 \mathrm{G}$, por lo que ha desplegado más de 600.000 unidades de antenas activas Massive 5G (AAU), posicionándose por encima de Samsung, Nokia, Ericsson y Qualcomm, empresas que también desarrollan tecnología 5G en el mercado (BBC, 2019; Contreras, 2020; EuropaPress, 2020).

Debido a esta escalada de la multinacional Huawei ${ }^{6}$, la empresa se encuentra en una posición privilegiada para liderar al mundo en el lanzamiento de un potencial cambio en la infraestructura tecnológica. Esto representa un riesgo para los Estados que históricamente han concentrado gran parte de este mercado. El que una empresa china sea pionera en la tecnología e infraestructura $5 \mathrm{G}$ implica que su influencia en el sistema internacional va a aumentar, por lo que tendrá una posición de ventaja sobre los demás a la hora de poder imponer sus intereses económicos, políticos y hasta culturales. El protagonismo e influencia de las potencias que antes ocupaban esta posición privilegiada en el sistema internacional se vería disminuido, sin contar con los efectos colaterales en el manejo de los flujos de información.

Adicional, el nuevo resurgir del Estado chino no ha sido bien recibido e incluso ha sido molesto para varios países en occidente, especialmente para Estados Unidos, el cual ve en la multinacional Huawei, un símbolo de competencia directa que puede condicionar su poderío en el sistema internacional. El Gobierno estadounidense ha puesto en tela de juicio la confiabilidad de esta empresa, aludiendo a su cercanía con

6 Que es coherente con el ascenso de la República Popular de China, que lleva 30 años de ascenso económico y de influencia en el orden internacional. 
el Gobierno chino y afirmando que la implementación de la tecnología $5 \mathrm{G}$ en cabeza de Huawei implica un tema de seguridad nacional, no solo para los Estados Unidos, sino para todos los Estados del orbe que se involucren con la tecnología 5G, ya que la dependencia tecnológica puede condicionar su futuro.

Este argumento estadounidense descansa en tres pilares: 1) la confrontación sistémica, 2) la seguridad y, 3) la economía (Rühlig et al. 2019). En el primer pilar, Estados Unidos percibe la rivalidad sobre la infraestructura 5G como parte de una confrontación de sistemas ${ }^{7}$. Luego de ocupar por décadas una posición privilegiada en el sistema internacional, disfrutando de los frutos del liberalismo económico, Estados Unidos debe adoptar una postura diferente a la del discurso empleado anteriormente, con la intención de que su posición no se vea vulnerada en el sistema internacional (Merino, 2019). Esto se evidenció en el periodo presidencial de Donald Trump (20172021), en el que el Estado norteamericano demostró orientar su política exterior a un proteccionismo cada vez más creciente. Con el lema "América first", el mandatario defendió las empresas estadounidenses de cualquier industria exterior que pudiera poner en riesgo su preponderancia, refiriéndose principalmente al mercado chino (Abella et al. s.f.). Asimismo, el actual presidente, Joe Biden, ha demostrado alinearse con esta postura, prohibiendo las inversiones estadounidenses en una decena de empresas de tecnología y defensa de China (BBC, 2021).

Por otro lado, China es una potencia que sigue en ascenso; presenta la característica especial de expresar una política de corte comunista-dictatorial dentro de su territorio, pero que al exterior implementa una economía de mercado (Singh, 2018). En los últimos años ha entablado relaciones amistosas con diferentes Estados, buscando dar a conocer su cultura e interfiriendo estratégicamente, en la medida de lo posible, dentro del marco político de otros Estados que consideren clave para la conformación de sus intereses (Abella et al. s.f.). Desde la óptica estadounidense, es en este punto en el que el mundo democrático liberal debe defenderse de la creciente influencia de la "autoritaria" nación China.

Como segundo pilar, en términos de seguridad, Estados Unidos afirma que Huawei podría ser utilizado por China para hacer espionaje político e industrial a través de su tecnología $5 \mathrm{G}$, señalando los antecedentes militares de Ren Zhengfei, fundador de Huawei, quien fue miembro del Ejército Popular de Liberación de las Fuerzas Armadas chinas, durante nueve años, hasta 1983, y que, además, es miembro del Partido Comunista de China (Bowler, 2020).

Desde la CIA hasta el presidente Donald Trump han señalado que la Ley de Inteligencia Nacional de China de 2017 (modificada en 2018), que establece que las compañías nacionales están obligadas a apoyar y cooperar en la red de inteligencia nacional del país, involucra directamente a Huawei con la comunidad de inteligencia y el Ejército chino, por lo que podría cooperar en un plan de vigilancia internacional a través de la tecnología nueva e innovadora del 5G (Fisher, 2019). De hecho, el caso planteado contra el gigante tecnológico chino por el Departamento de Justicia de EE. UU., donde le acusan de poner en marcha un esquema de crimen organizado y conspiración para

7 EE.UU sabe que China es un "rival estratégico": un socio comercial importante, pero también un adversario económico, político e ideológico (Cuenca, 2020). 
robar secretos comerciales nacionales, deja claro que los funcionarios estadounidenses creen que el éxito de Huawei se debe en parte a una política corporativa de espionaje y robo de propiedad intelectual (Guímon, 2020).

Por último, en cuanto al pilar tres, en un escenario económico en el que ambas potencias intentan ponderar su rol en el sistema internacional, Estados Unidos reúne esfuerzos para poder conservar su posición privilegiada en el sistema, conteniendo la escalada económica de China (Abella et al. n.d.). Las preocupaciones estadounidenses en este aspecto giran en torno al deseo de proteger las industrias nacionales de la "competencia desleal" y evitar el exceso de dependencia de la economía china, particularmente de su tecnología. Si bien los operadores estadounidenses podrían implementar la $5 \mathrm{G}$ más rápidamente con equipos chinos relativamente baratos, y obtener los beneficios indirectos al tener una red menos costosa, en un mercado que ya está muy concentrado ${ }^{8}$, esto podría restarle protagonismo, especialmente en el ámbito de las TIC $^{9}$, o implicar potencialmente preocupaciones de seguridad comercial (Brake, 2018; Rühlig et al., 2019).

Sin embargo, Estados Unidos no es el único que percibe la $5 \mathrm{G}$ a través de un lente geopolítico. China, por su parte, apunta a lograr una creciente cantidad de control sobre una amplia gama de flujos económicos a través del desarrollo de esta infraestructura en la zona euroasiática, intentando difundir su influencia en los países $\mathrm{BRI}^{10}$ por medio de la financiación, diseño y construcción de la infraestructura digital y física $5 \mathrm{G}$, que le ayuda a controlar los flujos de mercancías, servicios y datos (Rühlig et al., 2019). En ese sentido, Huawei es una empresa de importancia estratégica para ver realizados los intereses chinos. Esta empresa recibe tratamiento preferencial político, diplomático y sobre todo financiero. Para el año 2011, los préstamos blandos para Huawei ya ascendían a más de US \$ 30 mil millones, principalmente del Banco de Desarrollo (CDB), que es controlado por el Estado chino. Cabe resaltar que, de los 160.000 empleados de Huawei, 12.000 son miembros del Partido de Gobierno y forman no menos de 300 células partidarias dentro de la empresa (Rühlig y Björk, 2020). Esto evidencia el compromiso de parte del Gobierno chino por sacar este mercado adelante.

A la luz de estas consideraciones geopolíticas por parte de los Estados Unidos y China, se puede esperar que la $5 \mathrm{G}$ sea solo el primer capítulo en una competencia tecnológica, razonable, entre las dos políticas rivales, los cuales tienen como objetivo establecer, defender o ampliar sus esferas de influencia sectoriales y controlar los flujos de datos a través de alta tecnología.

\section{Acciones en torno al 5G en el escenario internacional}

Dicho lo anterior, podría entenderse por qué durante la última década Huawei ha sostenido una relación endeble con Estados Unidos. Como primer momento de tensión, se puede tomar la publicación en 2012 del Informe de seguridad "Investigative Report on the U.S. National Security Issues Posed by Chinese Telecommunications

\footnotetext{
8 Para junio de 2019 tan solo Samsung, Nokia, Ericsson, Qualcomm y Huawei manejaban el mercado de la tecnología 5G (BBC, 2019).

9 Tecnologías de la información y la comunicación.

10 Es la iniciativa de la Franja y la Ruta de la nueva ruta de seda (en inglés: Belt and Road Initiative, BRI), el cual pretende formar un conjunto de enlaces marítimos y ferroviarios entre China y Europa, pasando por Kazajistán, Rusia, Bielorrusia y Polonia, para terminar
} en Alemania, Francia y el Reino Unido. 
Companies Huawei and ZTE”, elaborado por la Comisión de Inteligencia de la Cámara de Representantes estadounidense. Esta investigación duro 11 meses y concluyó que los riesgos asociados con la provisión de equipos de Huawei y ZTE para la infraestructura crítica de los Estados Unidos, podrían ser determinantes en los intereses centrales de seguridad nacional, ya que no son claros sus nexos con el Gobierno chino. El informe sugiriere que Huawei no ha cumplido con las obligaciones legales de los Estados Unidos y las normas internacionales de conducta empresarial, y propone la prohibición del uso de su tecnología (García, 2012).

Del mismo modo, hace tres años, el primero de diciembre de 2018, al mismo tiempo que el expresidente estadounidense Donald Trump y su homólogo Xi Jinping se reunían en la Cumbre del G20 con la promesa de una tregua arancelaria de 90 días, la hija del fundador y vicepresidenta de Huawei, Meng Wanzhou, fue arrestada en Canadá en una solicitud de extradición de los Estados Unidos, acusada de "fraude bancario" y de haber violado las sanciones hacia Irán de parte de ese país. A pesar de que la heredera de Huawei, luego de varios días logró la libertad condicional, tras pagar una fianza por 10 millones de dólares canadienses, el Gobierno chino catalogó esta acción como una estrategia de parte de los Estados Unidos de América para desprestigiar y contener el ascenso económico de la compañía. (BBC, 2018; Vaswani, 2018).

Entre las acciones más recientes de Estados Unidos en contra de la empresa Huawei están las restricciones de las visas para sus empleados en EE. UU., luego de que la Comisión Federal de Comunicación (FCC) declarara el 30 de junio de 2019 a los gigantes chinos de las telecomunicaciones Huawei y ZTE como amenazas a la seguridad nacional. Adicional a esto, el gobierno de Donald Trump extendió la prohibición de usar equipos de telecomunicaciones de compañías como Huawei hasta mayo de 2021. La prohibición empezó a mediados de mayo de 2020, cuando Washington puso a la Huawei en una lista de entidades que no pueden comprar componentes electrónicos en Estados Unidos sin permiso de la administración. La medida fue suspendida durante 90 días a partir del 20 de mayo, pero las consecuencias de todas formas se vieron. Por ejemplo, la compañía Google anunció que dejaba de prestar servicios tecnológicos a la empresa china (Digitaltrends, 2020).

Dentro de esta misma línea de acciones, el presidente Joe Biden, a través de la Directiva presidencial que empezó a regir el 2 de agosto de 2021, amplía la orden ejecutiva que firmó en noviembre de 2020 su antecesor, Donald Trump. La Directiva presidencial pasa de vetar 30 compañías chinas a prohibir las inversiones de empresas estadounidenses y dar un plazo de un año para que se desprendan de los activos de 59 firmas del país asiático, incluyendo el gigante Huawei y las tres mayores compañías de telecomunicaciones de la nación: China Mobile, China Unicom y China Telecom, asegurando que la decisión busca impedir que se beneficie a compañías cuyas tecnologías de vigilancia contribuyen, dentro o fuera de China, al espionaje, además de facilitar la represión y graves abusos de derechos humanos (BBC. 2021).

Estas medidas también han sido apoyadas por gobiernos europeos. La Unión Europea insta a los Estados miembros a diversificar los proveedores en el despliegue de la quinta generación de redes móviles 5G. Algunos países, como Reino Unido, Francia y España, han prohibido o limitado el uso de los componentes Huawei, atribuyendo a cuestiones de soberanía y seguridad nacional. Para empezar, Reino Unido anunció 
que en 2021 prohibirá a los operadores de telecomunicaciones adquirir tecnología $5 G$ de Huawei, para garantizar la seguridad, tras las sanciones aplicadas a la empresa china por EE. UU.; España, por su parte, prohibió a sus funcionarios del Ministerio de Defensa conectarse a los sistemas de información del organismo desde los dispositivos fabricados por la firma china; por último, el Gobierno francés ha adoptado una postura más sutil, anunciado que se impondrán varias restricciones para las empresas que utilizan equipos chinos, sin que esto implique una prohibición total para Huawei (Digitaltrends, 2020; DW, 2020).

La República Popular de China, en respuesta a estas acciones, añadió en la lista de entidades poco confiables a las compañías estadounidenses Apple, Qualcomm y Cisco Systems. Alude China que las acciones por parte de Estados Unidos son injustas y niegan todas las acusaciones en cuanto al robo comercial y de espionaje del que se les ha señalado. El Gobierno chino ha afirmado que los países que arbitrariamente intenten atentar contra las empresas chinas tendrán represalias tanto económicas como políticas. Por su parte, el fundador de Huawei, Ren Zhengfei, ha dicho que estas prohibiciones, más que afectar a Huawei, lo que han hecho es perjudicar a las empresas estadounidenses que comercian con esta misma, por lo que ha ofrecido una solución lateral, prometiendo agrupar todas sus patentes $5 \mathrm{G}$, licencias, códigos y planos técnicos, para ponerlos a la venta en una transacción única. Según Zhengfei, la idea sería crear un rival para el gigante tecnológico chino, afirmando que "una distribución equilibrada de intereses es propicia para la supervivencia de Huawei" (Digitaltrends, 2020).

\section{Seguridad Nacional en tiempos de Big Data: Una óptica desde el "capitalismo de vigilancia” de Shoshana Zuboff}

En el relato de las tensiones entre las dos mayores potencias se puede pensar que todo se reduce a una discusión económica; pero más allá de esas consecuencias, se trata de mantener el control sobre los canales de información. La importancia de la red $5 G$ va más allá de la velocidad de carga y descarga en la telefonía móvil, tiene que ver sobre todo con el internet de las cosas y su infraestructura. El hecho no puede pasar desapercibido, pues la tecnología inteligente (desde un Smart tv, hasta un vehículo autónomo) recopila una cantidad de datos sin precedentes; a esto es lo que se denomina Big Data ${ }^{11}$ o macro datos. Esta cantidad de datos son los que pueden usarse para hacer perfilamiento comercial o político. Una vez hecha esa operación, predecir y manipular la opinión pública en una dirección o mantener el control de agentes molestos, se reduce a tan solo una operación de comandos en un servidor.

Para hacer este análisis es importante citar a la autora Shoshana Zubboff, quien en su libro "Surveillance capitalism", o como es su traducción al español Capitalismo de vigilancia, expone cómo las grandes plataformas sociales como Google y Facebook recolectan las experiencias privadas de los usuarios, desde datos de búsqueda, clics, hasta la permanencia en algún tipo de contenido en particular, los cuales se convierten en datos de comportamiento que luego pueden ser ofertados como productos en el

11 Zuboff sostiene que la "Big Data" son los datos o conjuntos de datos que se obtienen de las interacciones de los usuarios en la red. Los cuales se extraen de la vida de los usuarios sin su conocimiento o consentimiento informado (Zuboff, 2019). 
mercado (Mercados futuros) ${ }^{12}$. Para la autora, este tipo de recolección y comercio de datos se está gestando desde hace ya 20 años, pero es con el internet de las cosas (loT) que se hace más poderoso. A esta nueva lógica Zuboff la llama "Capitalismo de vigilancia", una nueva forma de capitalismo que predice las acciones de los usuarios a partir de los datos que ellos mismos producen y con los cuales es posible no solo predecir, sino incluso reorientar los hábitos de consumo o filiación a una campaña electoral puntual (Zuboff, 2019).

Por ende, se puede entender por qué la tecnología 5G toma tanta relevancia en el escenario internacional. Con la implementación de estas redes en cabeza de la tecnología china crece la preocupación por el posible robo de millones de datos, secretos industriales y datos Estatales. Una preocupación real, en tanto que la red $5 G$ supone una conectividad a escala de ciudad mayor a la actual, y al ser justamente Huawei quien tiene el mayor avance y la mayor cantidad de patentes en esta área de las telecomunicaciones, la implementación de la $5 G$ se convierte en una posible fuga de información hacia el gobierno de Xi Jinping, sumado a las preocupaciones respecto a China por espionaje corporativo, desde el robo de patentes intelectuales hasta el duplicado de ingeniería, junto con lo que se ha podido conocer de sus leyes de seguridad.

Las autoridades norteamericanas comprenden que la tecnología $5 G$ no acarrea en sí misma un riesgo, sin embargo, no es estratégico darle el control de su infraestructura tecnológica a quien pueda usar el flujo de macrodatos en su contra. La dependencia en sí misma de la tecnología $5 \mathrm{G}$, en un futuro próximo, tampoco es una carta que Estados Unidos quiera ceder.

Sumado a esto, se debe contemplar el riesgo que implicaría que una compañía china haga parte de la cadena de suministro de todas las entidades de los diferentes Estados que están haciendo una transición a la 5G. El proteccionismo estadounidense, cimentado desde la administración Trump, no está dispuesto a dar acceso a Pekín a todo el flujo de datos que ya manejan sus gigantes tecnológicos.

Como lo sostiene Zuboff (2019), la construcción de algoritmos de comportamiento a partir de la interacción de los usuarios en la red tiene la capacidad de cambiar la forma en que los seres humanos se comportan de manera individual y cómo interactúan con los de su propia especie y con su entorno, en razón a que no puede subestimarse su potencial de control y disciplina, ya sea para fines políticos o comerciales. Por lo anterior, las principales potencias del mundo están embarcadas en una lucha subyacente para asegurar su propio liderazgo para poseer el monopolio de la explotación y almacenamiento del Big Data que habilita la infraestructura 5G. Es el factor humano, y sus decisiones, la base fundamental de cualquier Estado. Quien logre persuadirlos, logra el dominio.

12 Estos datos de comportamiento se convierten en productos predictivos, los cuales, son comprados y vendidos en un nuevo tipo de mercado que Shoshana Zubboff denomina como "mercados de futuros conductuales", donde se comercializan algoritmos conductuales futuros de los usuarios (Zuboff, 2019). 


\section{Conclusiones}

China y Estados Unidos se encuentran en una disputa por liderar y controlar la implementación de la infraestructura 5G. Pero más allá de ser un problema netamente comercial, que tendrá repercusiones económicas, se convierte en un factor geopolítico y de seguridad nacional, que privilegiará a los Estados que sean pioneros en esta tecnología. El Estado que logre tener control de la tecnología 5G tendrá una ventaja a la hora de poder imponer sus intereses políticos, comerciales y hasta culturales a otro país, no solo por el grado de influencia y dependencia que genera en los demás, sino también por los datos y canales de datos a los que tendrá acceso a través de esta misma, convirtiéndose conceptualmente en un Estado con soberanía digital, al ser dueño de las patentes $5 \mathrm{G}$ y, por ende, de la investigación, los flujos de información y los datos que esta misma habilita, teniendo una independencia tecnológica frente a otros países, además de mantener una relación de interdependencia asimétrica con los demás Estados, quienes tendrían que ceder ante los intereses que ese país soberano digitalmente en $5 \mathrm{G}$ dicte.

Huawei se convierte en una pieza fundamental para el Gobierno de China al llevar la delantera en la tecnología 5G. Después de 30 años de ascenso, China se encuentra en una posición privilegiada para liderar al mundo en el cambio a una nueva infraestructura tecnológica, restándoles protagonismo a los países que antes ocupaban este lugar en el sistema internacional, en especial a Estados Unidos. Es por esto que este país categoriza a esta compañía china como un problema de seguridad nacional.

La desconfianza estadounidense sobre el Gobierno chino está cimentada en antecedentes de denuncias y sospechas sobre sus leyes de seguridad respecto a sus compañías, por lo que la posibilidad de la filtración de datos y robo de estos no es una preocupación menor para Estados Unidos. La posibilidad de que China pueda empezar a usar el flujo de datos proveniente de las redes $5 \mathrm{G}$ que instale generaría una competencia a empresas norteamericanas que ya hacen administración y comercio de datos; un área en que la Casa Blanca no quiere competencia para sus gigantes tecnológicos.

Sumado a esto, existe el interrogante del uso que podría darle el Gobierno chino a estos datos, puesto que, como lo sustenta Zuboff (2019), la mercantilización de las experiencias privadas de los usuarios, hechas por empresas como Google o Facebook, han demostrado que la tecnología, además de predecir, puede redireccionar el comportamiento comercial o la filiación política de los usuarios. Las personas no son conscientes de que sus hábitos, por mínimos que sean, generan dinero a conglomerados. Estos hábitos son datos que se convertirán en información que luego se monetizará y no beneficiará al individuo fuente.

Cada Estado teóricamente tiene normatividad para proteger a los individuos y sus datos, pero al ser usuario de empresas extranjeras y por temas de globalización, las normas quedan inoperantes. Realmente no hay forma de que exista "privacidad individual", a menos que una persona se aísle de la tecnología. Por lo tanto, el uso y el manejo de los flujos y canales de datos que habilita la infraestructura $5 \mathrm{G}$ se convierten en un recurso estratégico para los Estados. 


\section{Referencias}

Abella, G., Alemañy, P., Bas, E., Coggan, J y Mirazo, P. (s.f). Guerra Comercial China - Estados Unidos: Caso Huawei vs Estados Unidos [Archivo PDF]. https://www. academia.edu/41330718/Guerra_Comercial_entre_China_y_Estados_Unidos_ Caso_Huawei

Agencia AFP. (30 de julio de 2020). Huawei supera a Samsung y se convierte en líder de ventas de teléfonos. El Espectador. https://www.elespectador.com/noticias/ tecnologia/huawei-supera-a-samsung-y-se-convierte-en-lider-de-ventas-detelefonos/

BBC. (6 de diciembre de 2018). Huawei: Canadá arresta a Meng Wanzhou, directora financiera de la compañía, y China responde con enojo. BBC News. https://www. bbc.com/mundo/noticias-internacional-46465418

BBC. (7 de junio de 2019). Huawei: ¿qué empresas compiten con la compañía china en el desarrollo de la tecnología 5G?. BBC News. https://www.bbc.com/mundo/ noticias-48556359

BBC. (4 de junio de 2021). Estados Unidos vs China: la nueva orden de Biden contra empresas chinas que refuerza la dura posición de Washington con Pekín. BBC News. https://www.bbc.com/mundo/noticias-internacional-57364306

Bowler, T. (15 de julio de 2020). Huawei: por qué algunos países prohíben la tecnología $5 G$ del gigante chino y cuáles son los temores de espionaje. BBC News. https:// www.bbc.com/mundo/noticias-53413017

Brake, D. (2018). Economic Competitiveness and National Security Dynamics in the Race for $5 G$ between the United States and China. Journal Information Technology \& Innovation Foundation ITIF. https://doi.org/10.2139/ssrn.3142229

Cheng, R. (2 de marzo de 2017). Not just speed: 7 incredible things you can do with $5 \mathrm{G}$. CNET. https://www.cnet.com/es/noticias/redes-5g-velocidad-cosas-increiblesque-puedes-hacer/

CNN. (23 de mayo de 2019). 10 datos que muestran el alcance de Huawei en el mercado mundial. CNN Español. https://cnnespanol.cnn.com/2019/05/23/10-datos-quemuestran-el-alcance-de-huawei-en-el-mercado-mundial/

Contreras, V. (11 de junio de 2020). Huawei lidera las patentes 5 G declaradas y EE.UU. tendrá que pagar por esta tecnología. DPL News. https://digitalpolicylaw.com/ huawei-lidera-las-patentes-5g-declaradas-y-ee-uu-tendra-que-pagar-por-estatecnologia/

Cuenca, A. (28 de junio de 2020). El problema de Europa: la dependencia tecnológica de Estados Unidos y China. El Orden Mundial. https://elordenmundial.com/ dependencia-tecnologica-union-europea/ 
Digitaltrends. (9 de septiembre de 2020). Todo lo que deberías saber sobre la situación de Huawei. DT en español. https://es.digitaltrends.com/celular/huawei-androidgoogle-trump/

DW. (14 de julio de 2020). El Reino Unido excluye a Huawei del desarrollo de su red 5G. DW Made for minds. https://www.dw.com/es/el-reino-unido-excluye-a-huaweidel-desarrollo-de-su-red-5g/a-54172084

Europa Press. (20 de febrero de 2020). Huawei alcanza los 91 contratos comerciales relacionados con el 5G a nivel global. Europa Press. https://www.europapress.es/ economia/noticia-huawei-alcanza-91-contratos-comerciales-relacionados-5gnivel-global-20200220172629.html

Fisher, L. (20 de abril de 2019). CIA warning over Huawei. The Times. https://www. thetimes.co.uk/article/cia-warning-over-huawei-rz6xc8kzk

Flores, J. (28 de junio de 2019). Qué es el 5G y cómo nos cambiará la vida. National Geographic. https://www.nationalgeographic.com.es/ciencia/que-es-5g-y-comonos-cambiara-vida_14449

García, I. (2018). Cincuenta años de la Ofensiva del Tet: el punto de inflexión de la guerra del Vietnam. Revista del Instituto Español de Estudios Estratégicos, 782-800.

García, C. (8 de octubre de 2012). El Congreso de EE UU recomienda vetar a Huawei y ZTE. El País. https://elpais.com/tecnologia/2012/10/08/ actualidad/1349683984_930377.html

Gavaldá, J. (13 de julio de 2020). Chasquis, los mensajeros del Inca. National Geographic. https://historia.nationalgeographic.com.es/a/chasquis-mensajeros-inca_15459

Guímon, P. (14 de febrero de 2020). EE UU acusa a Huawei de espiar y robar tecnología a seis firmas estadounidenses. El País. https://elpais.com/economia/2020/02/13/ actualidad/1581620587_797527.html

Orellana, R. (2020). ¿Sabes qué es la red 5 G y qué ventajas nos ofrece?. Digitatrends ES. https://es.digitaltrends.com/celular/que-es-la-red-5g/

Merino, G. E. (2019). Guerra comercial y América Latina. Revista de Relaciones Internacionales de La UNAM (134), 67-98. ISNN 2448-8623.

Miller, R. (2 de julio de 2019). The 5G Rollout: The Hype is Building Faster Than the Infrastructure. Data Center Frontier. https://datacenterfrontier.com/the-5grollout-the-hype-is-building-faster-than-the-infrastructure/

Red Hat. (s.f.). What is IT infrastructure? https://www.redhat.com/es/topics/cloudcomputing/what-is-it-infrastructure

Rühlig, T. y Björk, M. (2020). What to Make of the Huawei Debate?5G Network Security and Technology Dependency in Europe. The swedish Institute of International Affairs. https://www.ui.se/globalassets/ui.se-eng/publications/ui-publications/2020/ ui-paper-no.-1-2020.pdf 
Rühlig, T., Seaman, J., \& Voelsen, D. (2019). 5G and the US-China tech rivalry - a test for Europe's future in the digital age : how can Europe shift back from back foot to front foot ?.German Institute for International and Security Affairs. https://doi. org/10.18449/2019C29

Singh, M. (2018). From Smart Power to Sharp Power: How China Promotes her National Interests. Journal of Defence Studies, 12(3), 5-25.

Torra, V. (2020). La inteligencia artificial. Cuadernos de la Fundación General CSIC. http://www.fgcsic.es/lychnos/es_es/articulos/inteligencia_artificial

Vaswani, K. (8 de diciembre de 2018). Arresto de Meng Wangzhou: ¿Se ha desatado una nueva guerra tecnológica entre Estados Unidos y China?. BBC News. https:// www.bbc.com/mundo/noticias-internacional-46491110

Zuboff, S. (2019). The Age of Surveillance Capitalism. Editorial: PublicAffairs 